Vietnam Journal of Mechanics, VAST, Vol.42, No. 4 (2020), pp. 363-374

DOI: https://doi.org/10.15625/0866-7136/14874

\title{
DESIGN AND ANALYSIS OF A DISPLACEMENT SENSOR-INTEGRATED COMPLIANT MICROGRIPPER BASED ON PARALLEL STRUCTURE
}

\author{
Nhat Linh $\mathrm{Ho}^{1}$, Minh Phung Dang ${ }^{2}$, Thanh-Phong Dao ${ }^{3,4, *}$ \\ ${ }^{1}$ Koei Vietnam Company Limited, Ho Chi Minh City, Vietnam \\ ${ }^{2}$ Faculty of Mechanical Engineering, Ho Chi Minh City University of Technology \\ and Education, Ho Chi Minh city, Vietnam \\ ${ }^{3}$ Division of Computational Mechatronics, Institute for Computational Science, \\ Ton Duc Thang University, Ho Chi Minh city, Vietnam \\ ${ }^{4}$ Faculty of Electrical \& Electronics Engineering, Ton Duc Thang University, \\ Ho Chi Minh city, Vietnam \\ *E-mail: daothanhphong@tdtu.edu.vn
}

Received: 08 March 2020 / Published online: 17 August 2020

\begin{abstract}
This study evaluates the displacement sensitivity of a new compliant microgripper. The microgripper is designed based on a four-bar mechanism and the concept of a compliant mechanism. The effects of the width of the right circular hinge, the thickness of microgripper, and the material properties on the dis-placement sensitivity are considered via using the finite element method. In the beginning, the stress and deformation of the compliant microgripper are evaluated. Subsequently, the displacement of the microgripper is then analyzed. The results showed that the design parameter and the displacement sensitivity have a close relationship. To increase the grasping reliability and measure the displacement or force, a micro-displacement sensor is integrated with the proposed microgripper. Finally, the modeling and analysis of the proposed sensor are conducted.
\end{abstract}

Keywords: displacement sensor, microgripper, parallel structure, FEM, four-bar mechanisms.

\section{INTRODUCTION}

Take many advantages of compliant mechanisms such as monolithic, small size, high precision, and low cost, simple fabrication method [1,2], compliant microgripper has become vitally necessary devices and widely used in the industry. Especially, the industries include biomedical engineering, micro assembling engineering, manipulation of microobjects, medical application, and high precision manipulation application $[3,4]$. In the last few years, the compliant mechanisms have received considerable attention by Huang and Dao [5], Royson et al. [6], Hao et al. [7], Chen et al. [8], Ho et al. [2]. The application 
of compliant mechanisms to microgripper has been made with many different types. The microgripper with double rocker mechanism and the parallelogram mechanism was proposed by Yang et al. [9], a compliant gripper using an optimal Scott-Russell mechanism was designed by Ai et al. [10], etc.

To grasp and release the object precisely as well as avoid distorting the object, a displacement sensor, which can apprehend the motion range of micrometer, is needed for the gripper. Recently, integrating a displacement sensor into the gripper was designed by Shafaat et al. [11] and Yang et al. [12]. Moreover, to ensure reliable and effective performances for compliant microgripper during the grasping process, the stress, displacement, vibration, buckling, failure, and optimization were simultaneously considered $[5,10,13,14]$. However, an evaluation of the displacement sensitivity of the compliant microgripper has been less interested.

The goal of this paper is to design and evaluate the displacement sensitivity of the compliant microgripper. A micro-displacement sensor is integrated monolithically with the proposed microgripper to measure the displacement or force during the grasping process. A parallel structure of the four-bar mechanism is used for designing the microgripper. The gripper is designed to have a positioning range of more than $500 \mu \mathrm{m}$ for flexibility in use. A finite element analysis is conducted to investigate static performances. Effect of the width of the right circular hinge $(\mathrm{RCH})$, the thickness of the gripper, and the material properties on the displacement sensitivity are carried out. Eventually, modeling and simulation for the proposed sensor are analyzed.

\section{COHERENCE OF STRUCTURE}

The microgripper was developed based on the four-bar mechanism, as indicated in the Fig. 1(a). Also, to increase the firmness of the model, at the position of bar 1 and bar 3 were reinforced by a similar bar, as displayed in the Fig. 1(b).

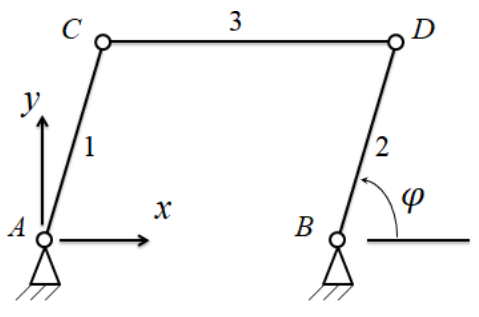

(a) Simple four-bar mechanisms

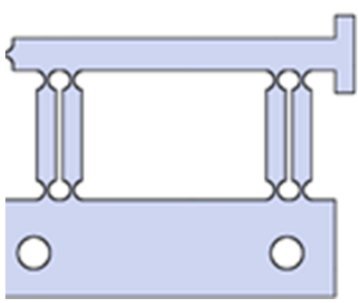

(b) Four-bar mechanisms after reinforcement

Fig. 1. Four bar mechanisms

For the purpose, the compliant microgripper must have the minimized operating errors; the circular hinge was hence selected for constructed a microgripper because the rotation center of the circular hinge was almost unchanged throughout the operation [15]. The model of the circular hinge was illustrated in Fig. 2 and the spring rate of the circular 
hinge can be calculated by [16]

$$
k_{\theta}=\frac{2 E t w^{2.5}}{9 \pi R^{0.5}}
$$

where $k_{\theta}, E, t, w, R$, are the angular stiffness, Young's modulus of the material, the thickness of the full $\mathrm{RCH}$ (thickness of model), the width of $\mathrm{RCH}$, and radius of the hinge, respectively.

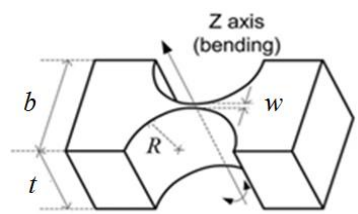

Fig. 2. Right circular hinge

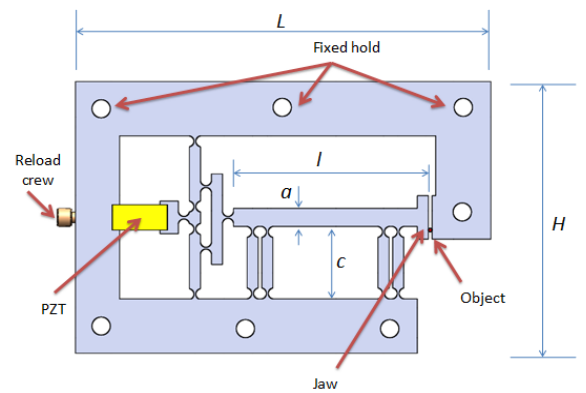

Fig. 3. Schematic diagram of the microgripper

From Eq. (1), it is noted that the stiffness is related to the geometrical parameters of the structure. As known, the stiffness is inversely proportional with the compliance of the microgripper by the following equation [17]

$$
C=\frac{d}{F}=\frac{1}{k}
$$

where $C, d, F, k$ are the compliance, displacement, load, and stiffness of the microgripper, respectively.

Besides, Eq. (2) also implies that the displacement is proportional to the compliance. To sum up, a change in each of the geometrical parameters, the displacement of the gripper is varied. Hence, the sensitivity of displacement is dependent on the design parameters. Besides, the displacement also depends on the used materials.

There are a lot of various types of materials that can be used for the compliant microgripper such as aluminum alloy, titanium alloy, structure steel, etc. The compliant microgripper must have a compact in size and a light in weight. Regarding the geometrical parameters of the proposed microgripper, polyethylene was chosen because it was relatively light with good mechanical properties as the Yield strength of $25 \times 10^{-3} \mathrm{GPa}$, Density $\rho$ of $950 \mathrm{~kg} / \mathrm{m}^{3}$ and Young's modulus of $1.1 \mathrm{GPa}$.

Based on the above suggestions, the compliant microgripper was constructed as in Fig. 3 with details as follows: (i) Seven fixed holes were used to locate the gripper while test it characteristics, (ii) a piezoelectric actuator (PZT) was used to exert the force to the gripper through a linear mechanism connected with a four-bar mechanism, (iii) microsized objects would be grasped by a fixed jaw and movable jaw. The total dimension of the model was about $75 \mathrm{~mm} \times 113 \mathrm{~mm} \times 5 \mathrm{~mm}$. The parameters of the microgripper were given in Tab. 1. The proposed gripper was designed to handle micro-sized objects of $500 \mu \mathrm{m}$. 
Table 1. The size of the compliant microgripper

\begin{tabular}{clc}
\hline Symbol & \multicolumn{1}{c}{ Description } & Value $(\mathrm{mm})$ \\
\hline$L$ & Length of model & 113 \\
$H$ & Height of model & 75 \\
$w$ & Width of a circular hinge & 0.5 \\
$l$ & Length of bar & 28.5 \\
$t$ & Thickness of circular hinge & 5 \\
$R$ & Radius of circular hinge & 1.53 \\
$a$ & Width of bar & 5 \\
$b$ & Width of bar & 3 \\
$c$ & Height of four-bar mechanism & 20 \\
\hline
\end{tabular}

Total dimension: $75 \mathrm{~mm} \times 113 \mathrm{~mm} \times 5 \mathrm{~mm}$

\section{FINITE ELEMENT ANALYSIS}

The finite element analysis (FEA) in ANSYS 16.0 was adopted to predict the output displacement, stress distribution, and displacement sensitivity of the gripper. To achieve this analysis, the automatically meshing method was applied, and hereafter each $\mathrm{RCH}$ was refined to achieve analysis accuracy. Also, the boundary conditions were proposed as follows: the gripper was fixed at the holes and the force's PZT was changed from $1 \mathrm{~N}$ to $7 \mathrm{~N}$. For evaluation on displacement sensitivity, the force of $2 \mathrm{~N}$ would be applied to the PZT. With the force provided from the PZT actuator, the jaw of microgripper was moved along the $z$-axis and pick up the object. Under subjecting this drive, the microgripper was deformed. The stress distribution and the deformation can be seen in Figs. 4 and 5 . Through these figures, it can be concluded that the maximum stress is at the middle of the circle hinges.
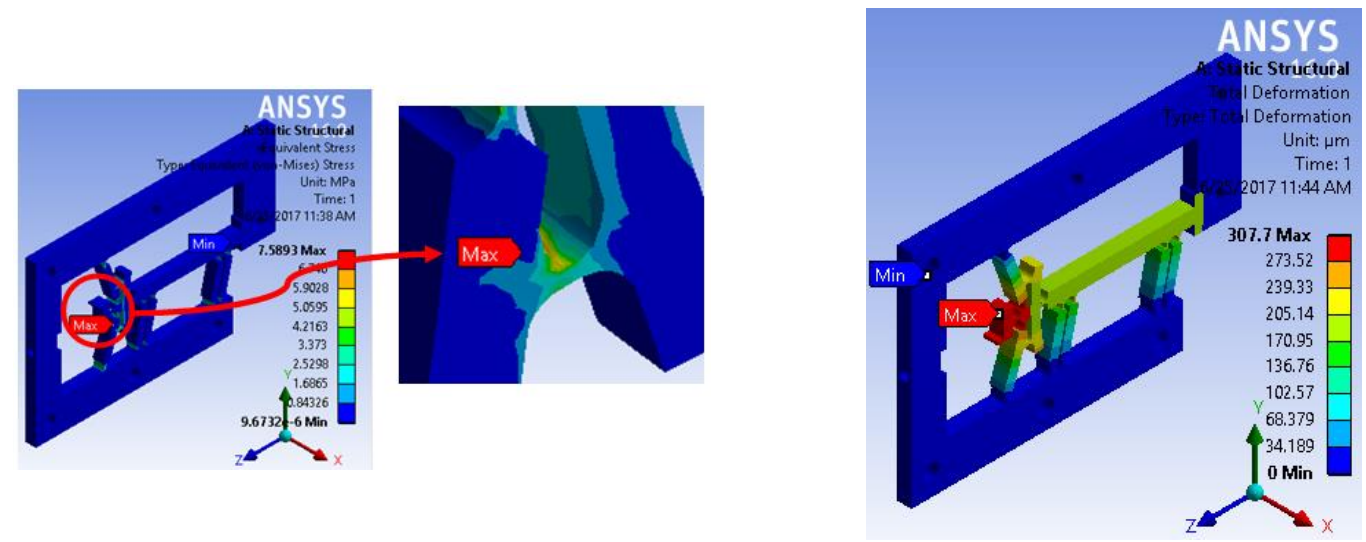

Fig. 4. Stress distribution of the compliant gripper Fig. 5. Deformation of the compliant gripper 


\section{RESULTS AND DISCUSSION}

\subsection{Analysis of the maximum displacement of the gripper}

The model of microgripper with parameters as Tab. 1 was used. The actuator force of $2 \mathrm{~N}$ would be applied to the PZT. The analysis process was performed by increasing the actuator force from $1 \mathrm{~N}$ to $7 \mathrm{~N}$, respectively. Polyethylene was selected for this analysis. The results were shown in Tab. 2.

Table 2. The output displacement

\begin{tabular}{ccc}
\hline Force $(\mathrm{N})$ & Displacement $[\delta-\mu]$ & Stress $\times 10^{-3}(\mathrm{GPa})$ \\
\hline 1 & 90.82 & 3.79 \\
2 & 181.64 & 7.59 \\
3 & 272.46 & 11.38 \\
4 & 363.29 & 15.18 \\
5 & 454.11 & 18.97 \\
6 & 544.93 & 22.70 \\
7 & 635.75 & 26.56 \\
\hline
\end{tabular}

The results in Tab. 2 indicated that when the actuator force is $7 \mathrm{~N}$, then the displacement of the jaw reaches the position of $635.75 \mu \mathrm{m}$ and the corresponding stress value is $26.56 \times 10^{-3} \mathrm{GPa}$. This value of stress was greater than the critical stress of the material $\left(25 \times 10^{-3} \mathrm{GPa}\right)$, the gripper could be a failure. Hence, the value of $544.93 \mu \mathrm{m}$ was selected as the maximum value of output displacement corresponding to the force of PZT was $6 \mathrm{~N}$ and the value of stress was $22.70 \times 10^{-3} \mathrm{GPa}$.

\subsection{Evaluation of displacement sensitivity of a compliant microgripper}

A relationship between the design parameters of microgripper as the material properties, the width of the circular hinge, and the thickness of the microgripper with the displacement sensitivity was investigated. Based on these results, the design parameters can be adjusted to create a reasonable design.

At present, some methods can be used to calculating the sensitivity as Nelson method, Modal method, matrix perturbation method, a differential method [18]. In this study, the direct differential method is chosen to use. And the relationship between the displacement sensitivity and the design parameter can be described as follows

$$
S_{D}=\frac{\partial D}{\partial x}
$$

where $S_{D}, D, x$ are the sensitivity, displacement and design parameters, respectively.

\subsection{Evaluate effect of the material properties on the value of displacement sensitivity}

The relationship between the material properties of the value of displacement was investigated. The density $(\rho)$ of material and the elastic modulus $(E)$ of material were chosen as design variables while other design parameters were proposed, as in Tab. 1. 
The investigation was conducted with four types of materials such as: Polyethylene $\left(\rho=950 \mathrm{~kg} / \mathrm{m}^{3}, E=1.1 \mathrm{GPa}\right)$, Structural steel $\left(\rho=7850 \mathrm{~kg} / \mathrm{m}^{3}, E=200 \mathrm{GPa}\right)$, Aluminum alloy $\left(\rho=2770 \mathrm{~kg} / \mathrm{m}^{3}, E=71 \mathrm{GPa}\right)$ and Titanium alloy $\left(\rho=4620 \mathrm{~kg} / \mathrm{m}^{3}\right.$, $E=96 \mathrm{GPa})$. The relationship between displacement sensitivity and design variables was determined through Eq. (3). The results were given in Tab. 3.

Table 3. Effect of the material properties to the value of displacement sensitivity

\begin{tabular}{lccc}
\hline \multirow{2}{*}{ Material } & Displacement & \multicolumn{2}{c}{ Displacement sensitivity } \\
\cline { 3 - 4 } & $(\mu \mathrm{m})$ & $S E=D / E(\mu \mathrm{m} / \mathrm{GPa})$ & $S \rho=D / \rho\left(\mu \mathrm{m} / \mathrm{kg}^{\left.-\mathrm{m}^{-3}\right)}\right.$ \\
\hline Polyethylene & 181.64 & 165.12 & 0.19 \\
Structural steel & 1.0728 & 0.005 & 0.0001 \\
Aluminum alloy & 2.1716 & 0.02 & 0.0004 \\
Titanium alloy & 2.9826 & 0.04 & 0.001 \\
\hline
\end{tabular}

Tab. 3 showed that the value of displacement sensitivity is inversely proportional to the elastic modulus of the material. The value of displacement sensitivity of the gripper reached to maximum when fabricated from Polyethylene material ( $E=1.1 \mathrm{GPa})$ and minimal when fabricated from Structural steel material $(E=200 \mathrm{GPa})$. This means that in order to increase the displacement sensitivity of the gripper, the lower elastic modulus should be selected. However, for the density of the material, its effect on the displacement sensitivity was not following any rules.

\subsection{Evaluate effect of the thickness on the value of displacement sensitivity}

All of the design parameters of the gripper remained unchanged from Tab. 1. However, the thickness of the microgripper model was changed to $4 \mathrm{~mm}, 5 \mathrm{~mm}$, and $6 \mathrm{~mm}$, respectively. Polyethylene was selected as fabricating material of this microgripper, which has the following properties: Yield strength of $25 \times 10^{-3} \mathrm{GPa}$, the density of $\rho=$ $950 \mathrm{~kg} / \mathrm{m}^{3}$, and Young's modulus of $1.1 \mathrm{GPa}$. The analysis results were shown in Tab. 4 .

Table 4. Effect of the thickness on the displacement sensitivity

\begin{tabular}{ccc}
\hline Thickness $[t-\mathrm{mm}]$ & Displacement $[D-\mu \mathrm{m}]$ & Displacement Sensitivity $S_{t}=D / t[\mathrm{~mm} / \mu \mathrm{m}]$ \\
\hline 4 & 230.23 & 57.557 \\
5 & 181.64 & 36.328 \\
6 & 151.85 & 25.308 \\
\hline
\end{tabular}

The results show that the value of displacement sensitivity of the gripper increases when the thickness of $\mathrm{RCH}$ decreases and inversely. In more detail, the $S_{t}$ was $57.557 \mathrm{~mm} / \mu \mathrm{m}$, when $t=4 \mathrm{~mm}$, the $S_{t}$ was $36.328 \mathrm{~mm} / \mu \mathrm{m}$ when $t=5 \mathrm{~mm}$ and the $S_{t}$ was $57.557 \mathrm{~mm} / \mu \mathrm{m}$ when $t=6 \mathrm{~mm}$. It means that, when designing a microgripper, increasing or decreasing the thickness of the microgripper model should be considered. 


\section{ANALYSIS FOR INTEGRATED SENSOR}

To grasp and locate the object precisely to avoid distorting the object. A displacement sensor is needed should be integrated for the microgripper. In the following, the structure of the microgripper with integrated displacement sensors will be proposed.

\subsection{Description of mechanical design}

The structure is a combination of two modules displacement sensor and microgripper, which was introduced in section second, as depicted in Fig. 6. The sensor's topology has a symmetrical structure based on the combination of a flexure-based micropositioning platform and strain gauges, as illustrated in Fig. 7. And the flexure-based micro-positioning platform is developed by a combination of sixteen flexure hinge with a rigid link. This structure allows for a large displacement range and flexibility.

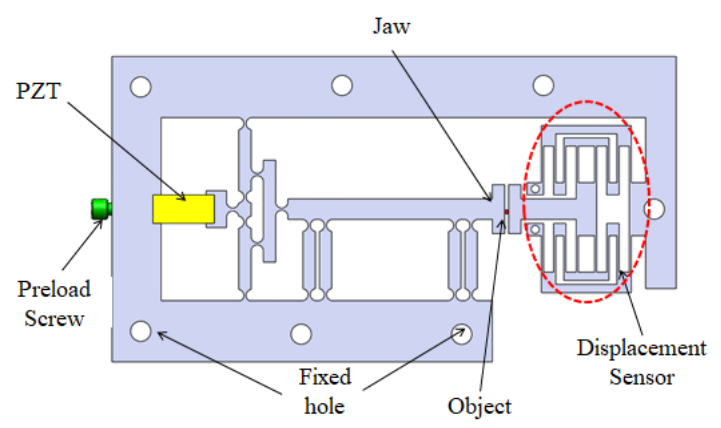

Fig. 6. A compliant microgripper with sensor force

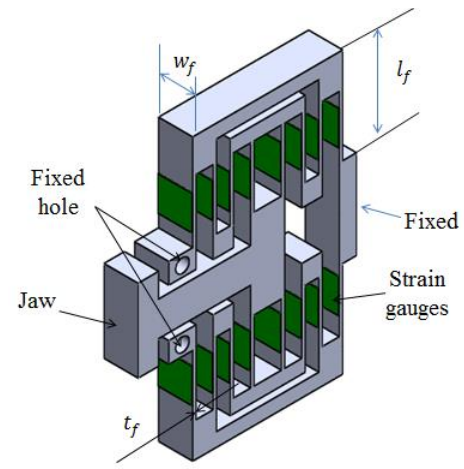

Fig. 7. Schematic diagram of displacement sensor

\subsection{The principle of operation of the displacement sensor}

Based on the combination of flexure hinge and strain gauges, the displacement sensor is created, as shown in Fig. 7. Here, a half-Wheatstone bridge circuit is recommended. Fig. 8 shows in the compressed state, the resistance $(R)$ decreases with the amount of $\Delta \mathrm{R}$ and vice versa, in the tension state, resistance $(R)$ will increase the amount of $\Delta R$.

And according to [19], the gauges factor of strain gauges are defined as follows

$$
G=\frac{\Delta R}{R \varepsilon}
$$

where $G, \Delta R, R$, and $\varepsilon$ are the gauge factor of the strain gauge, change the value of the gauge resistance, the nominal value of the gauge strain as an un-deformed strain gauge and nominal strain, sequentially.

Also, the relationship between nominal strain and stress is defined as follows

$$
\sigma=E \varepsilon,
$$

where $\sigma$ is the stress and $E$ is Young's modulus of the material. In this study, the proposed material was used Polyethylene. 


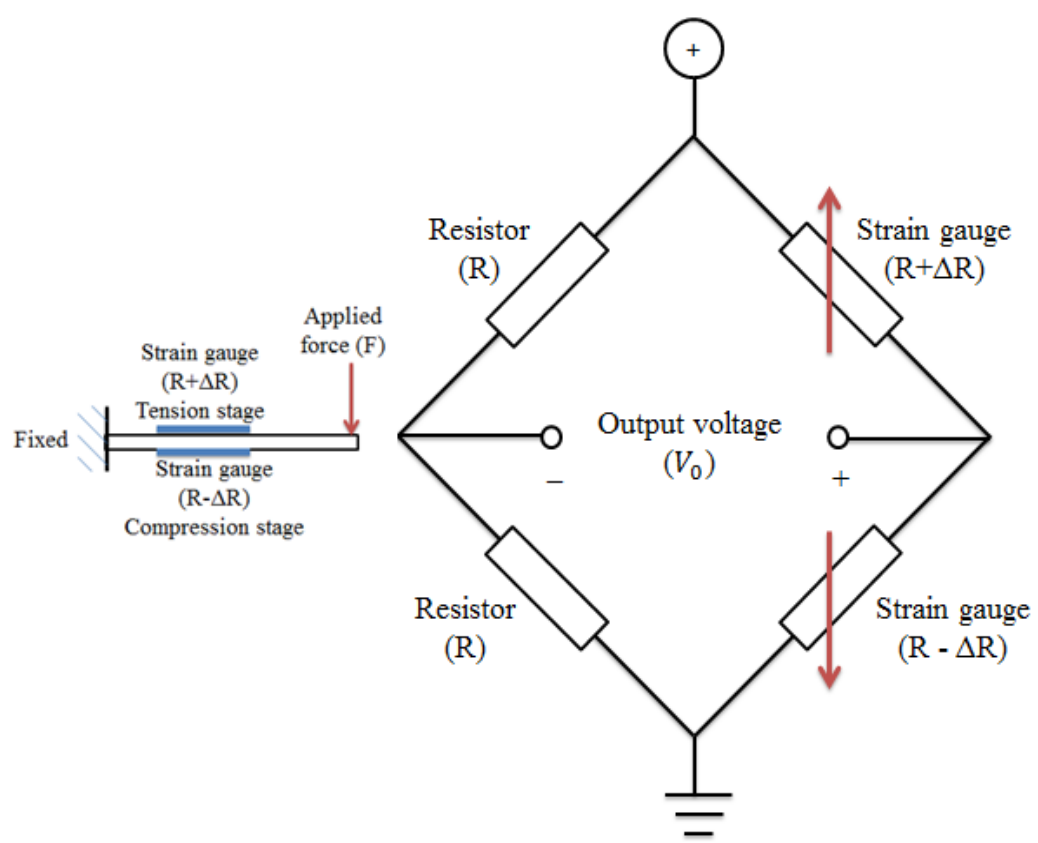

Fig. 8. Half-Wheatstone bridge circuit of the strain gauge

And the relationship between the input voltage and output voltages is approximated as follows

$$
V_{0}=\frac{G \varepsilon}{2} V_{e x}=\frac{\Delta R}{2 R} V_{e x},
$$

where $V_{0}$ is the output voltage of the bridge, and $V_{e x}$ is the excitation voltage.

From Eqs. (4)-(6), the relationship between the input voltage and output voltages can be rewritten as follows

$$
V_{0}=\frac{G \sigma}{2 E} V_{e x}
$$

Eq. (7) implies that the output voltage is directly proportional to the stress of the material.

Because the structure of the displacement sensor is symmetric and is constructed from many similar flexure hinges. So, in this study, only one flexure hinge element is selected for mathematical analysis.

Based on the cantilever beam theory [1], the linear stiffness of the flexure hinge is calculated as follows

$$
K_{A}=\frac{1}{4} \frac{E w_{A} t_{A}^{3}}{l_{A}^{3}},
$$

where $K_{A}$ is the stiffness of the flexure hinge $A ; w_{A}$ is the width of flexure hinge $A ; t_{A}$ is the thickness of flexure hinge $A$ and $l_{A}$ is the length of flexure hinge $A$. 
Also, the relationship between the force $(F)$ and the displacement $(\delta)$ of the flexure hinge by is expressed by the following Eq. (9)

$$
F=K_{A} \delta \text {. }
$$

The relationship between the force $(F)$ and stress is expressed by the following equation

$$
\sigma=\frac{6 F l_{A}}{w_{A} t_{A}^{2}} .
$$

By rearranging Eqs. (7)-(10), obtaining the Eq. (11)

$$
V_{0}=\frac{3}{4} \frac{V_{e x} G t_{A}}{l_{A}^{2}} \delta
$$

Eq. (11) can be rewritten as follows

$$
V_{0}=S \delta
$$

where $S$ is called the sensitivity coefficient of the flexure hinge or the sensitivity of the displacement sensor

$$
S=\frac{3}{4} \frac{V_{e x} G t_{A}}{l_{A}^{2}}
$$

Ultimately, by combining Eq. (5) and Eq. (10) obtaining Eq. (14)

$$
\varepsilon=\frac{6 F l_{A}}{E w_{A} t_{A}^{2}}
$$

Eq. (14) indicates that the value of the nominal strain is directly proportional to external forces $(F)$, the length of flexure hinge, and inversely proportional to the thickness of flexure hinge, the width of flexure hinge, and the coefficient Young's modulus of the material.

In short, the results of the analysis showed that sensitivity $(S)$ of the displacement sensor can be determined by determining the output displacement of the platform and the output voltage. And the value of sensitivity is influenced by factors such as the excitation voltage, the gauge factor, and the geometric parameters of the platform (Eq. (13)). Moreover, the results also show that the output voltage has a linear relationship with the displacement of the platform (Eq. (11)). As well as, the flexure hinge's ability to deform depends on its geometry parameters (Eq. (14)).

\subsection{The position of strain gauges}

To be able to accurately forecast the displacement value, it is necessary to put strain gauges in place. In this section, via finite element software ANSYS, appropriate strain gauges placement will be found. To accomplish this, first through the Solidworks software the model of the displacement sensor is constructed. In this study, $9 \times 5 \times 0.5 \mathrm{~mm}$ flexure hinge is used. Hereafter, the automatically meshing method was applied, and to achieve analysis accuracy each flexure hinge was refined, as shown in Fig. 9. Eventually, some of the assumptions are set up such as input displacement of $300 \mu \mathrm{m}$, and polyethylene was chosen as the material for the model.

Through FEA simulation, the maximum stress, the maximum strain, and the maximum displacement of the sensor are obtained $1.3634 \mathrm{MPa}, 1.24 \times 10^{-3} \mu \mathrm{m}$, and $300 \mu \mathrm{m}$, 
respectively. And stress distribution, strain distribution, and displacement distribution are illustrated as Figs. 10, 11, and 12, respectively.

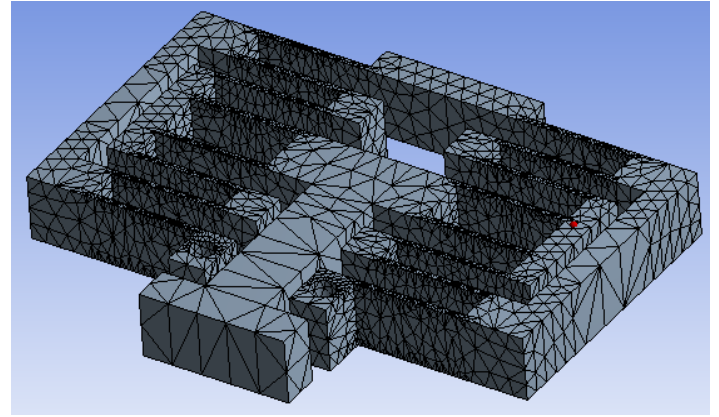

Fig. 9. Meshed model of the displacement sensor

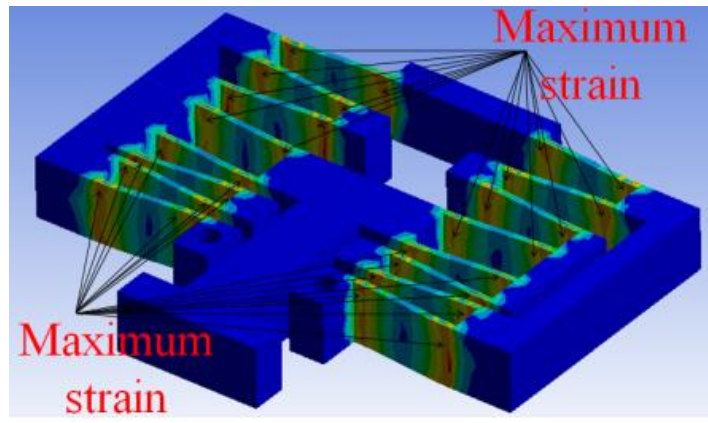

Fig. 11. Strain distribution of sensor

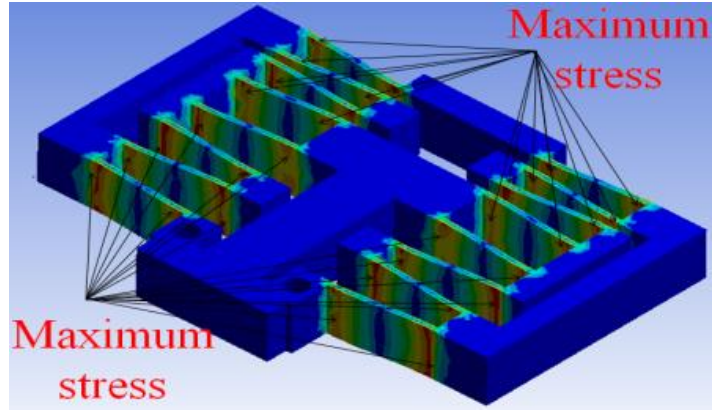

Fig. 10. Stress distribution of sensor

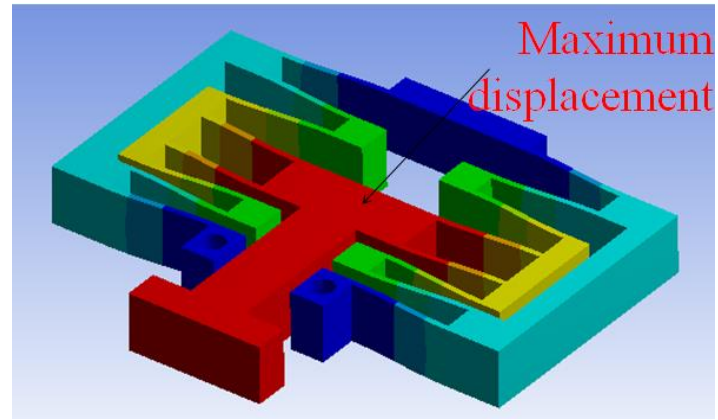

Fig. 12. Displacement distribution of sensor

Fig. 10 and Fig. 11 shows that the value of stress and the values of strain are maximized at the two endpoints of flexure hinge. And, the value of stress and the values of strain are maximized in the middle of the flexure hinge. Therefore, the two endpoints of the flexure hinge are defined as the best sensitivity for the sensor. This implies that Strain gauges should be attached at the two ends of the flexure hinge. It also found that the maximum displacement value of the sensor is on the jaw.

The strain gauges can be glued to the top surface or bottom surface of a flexure hinge or both. However, the results obtained are the same in value. It means that the strain in the tension state and the strain in the compression state are equal but the strain in the tension state has a positive value and vice versa, the strain in the compression tension state has a negative value.

\section{CONCLUSIONS}

In this article, the compliant microgripper was designed based on a four-bar mechanism and the right circular hinge. Then, the effect of the design parameters and the 
material properties on the displacement sensitivity of the compliant microgripper were analyzed by FEA. The results obtained as follows: The thickness of the microgripper was inversely proportional to the displacement sensitivity, the width of the $\mathrm{RCH}$ was inversely proportional to the displacement sensitivity. The material properties also had a close relationship with the displacement sensitivity. Therefore, these parameters should be considered when designing. Also, to increase the reliability and efficiency of the gripper, a displacement sensor is integrated into the gripper.

In future work, the frequency sensitivity, modeling, analytical analysis, kinematics, dynamics, and optimization for multi-performances of the proposed microgripper will be taken into account. A prototype will be fabricated for experimental testing and verify the FEA results. And then a micro-displacement sensor will be integrated monolithically with the proposed micro-gripper.

\section{ACKNOWLEDGMENT}

This research is funded by the Vietnam National Foundation for Science and Technology Development (NAFOSTED) under grant number 107.01-2019.14.

\section{REFERENCES}

[1] K.-B. Choi and D.-H. Kim. Monolithic parallel linear compliant mechanism for two axes ultraprecision linear motion. Review of Scientific Instruments, 77, (6), (2006), pp. 1-7. https://doi.org/10.1063/1.2207368.

[2] N. L. Ho, T.-P. Dao, N. Le Chau, and S.-C. Huang. Multi-objective optimization design of a compliant microgripper based on hybrid teaching learning-based optimization algorithm. Microsystem Technologies, 25, (5), (2019), pp. 2067-2083. https://doi.org/10.1007/s00542-0184222-6.

[3] M. C. Carrozza, A. Eisinberg, A. Menciassi, D. Campolo, S. Micera, and P. Dario. Towards a force-controlled microgripper for assembling biomedical microdevices. Journal of Micromechanics and Microengineering, 10, (2), (2000), pp. 271-276. https://doi.org/10.1088/0960$1317 / 10 / 2 / 328$.

[4] N. L. Ho, T.-P. Dao, H. G. Le, and N. Le Chau. Optimal design of a compliant microgripper for assemble system of cell phone vibration motor using a hybrid approach of ANFIS and Jaya. Arabian Journal for Science and Engineering, 44, (2), (2019), pp. 1205-1220. https://doi.org/10.1007/s13369-018-3445-2.

[5] T.-P. Dao, N. L. Ho, T. T. Nguyen, H. G. Le, P. T. Thang, H.-T. Pham, H.-T. Do, M.D. Tran, and T. T. Nguyen. Analysis and optimization of a micro-displacement sensor for compliant microgripper. Microsystem Technologies, 23, (12), (2017), pp. 5375-5395. https://doi.org/10.1007/s00542-017-3378-9.

[6] R. D. Dsouza, K. P. Navin, T. Theodoridis, and P. Sharma. Design, fabrication and testing of a 2 DOF compliant flexural microgripper. Microsystem Technologies, 24, (9), (2018), pp. 38673883. https://doi.org/10.1007/s00542-018-3861-y.

[7] G. Hao and R. B. Hand. Design and static testing of a compact distributed-compliance gripper based on flexure motion. Archives of Civil and Mechanical Engineering, 16, (2016), pp. 708716. https://doi.org/10.1016/j.acme.2016.04.011.

[8] X. Chen, Z. Deng, S. Hu, J. Gao, and X. Gao. Design of a compliant mechanism based four-stage amplification piezoelectric-driven asymmetric microgripper. Micromachines, 11, (1), (2020). https://doi.org/10.3390/mi11010025. 
[9] Y.-L. Yang, Y.-D. Wei, J.-Q. Lou, G. Tian, X.-W. Zhao, and L. Fu. A new piezo-driven microgripper based on the double-rocker mechanism. Smart Materials and Structures, 24, (7), (2015), pp. 1-11. https://doi.org/10.1088/0964-1726/24/7/075031.

[10] W. Ai and Q. Xu. New structural design of a compliant gripper based on the Scott-Russell mechanism. International Journal of Advanced Robotic Systems, 11, (12), (2014), pp. 1623-1628. https://doi.org/10.5772/59655.

[11] S. A. Bazaz, F. Khan, and R. I. Shakoor. Design, simulation and testing of electrostatic SOI MUMPs based microgripper integrated with capacitive contact sensor. Sensors and Actuators A: Physical, 167, (1), (2011), pp. 44-53. https://doi.org/10.1016/j.sna.2010.12.003.

[12] S. Yang and Q. Xu. Design and simulation a MEMS microgripper with integrated electrothermal actuator and force sensor. In 2016 International Conference on Advanced Robotics and Mechatronics (ICARM), IEEE, (2016), pp. 271-276. https://doi.org/10.1109/icarm.2016.7606931.

[13] M. H. Niaki and A. Nikoobin. Design and fabrication a long-gripping-range microgripper with active and passive actuators. Iranian Journal of Science and Technology, Transactions of Mechanical Engineering, 43, (3), (2019), pp. 575-585. https://doi.org/10.1007/s40997-017-01358.

[14] P. H. Phu, N. N. Viet, N. M. Ngoc, V. N. Hung, and C. D. Trinh. Simulation and optimization of a silicon-polymer bimorph microgripper. Vietnam Journal of Mechanics, 34, (4), (2012), pp. 247-259. https://doi.org/10.15625/0866-7136/34/4/2339.

[15] S.-C. Huang and T.-P. Dao. Design and computational optimization of a flexurebased XY positioning platform using FEA-based response surface methodology. International Journal of Precision Engineering and Manufacturing, 17, (8), (2016), pp. 1035-1048. https://doi.org/10.1007/s12541-016-0126-5.

[16] R. S. Joshi, A. C. Mitra, and S. R. Kandharkar. Displacement Analysis of Rectangular and Circular Hinge for Compliant Micro-Gripper. IOSR Journal of Mechanical \& Civil Engineering, (206), pp. 44-48. https://doi.org/10.9790/1684-1500844-48.

[17] L. L. Howell. Compliant mechanisms. John Wiley \& Sons, Ltd, (2011).

[18] S. Liu, J. Dai, A. Li, Z. Sun, S. Feng, and G. Cao. Analysis of frequency characteristics and sensitivity of compliant mechanisms. Chinese Journal of Mechanical Engineering, 29, (4), (2016), pp. 680-693. https://doi.org/10.3901/cjme.2015.1215.148.

[19] T.-P. Dao, N. L. Ho, T. T. Nguyen, H. G. Le, P. T. Thang, H.-T. Pham, H.-T. Do, M.D. Tran, and T. T. Nguyen. Analysis and optimization of a micro-displacement sensor for compliant microgripper. Microsystem Technologies, 23, (12), (2017), pp. 5375-5395. https://doi.org/10.1007/s00542-017-3378-9. 